\title{
CORRECTION
}

\section{Correction to: Crack mathematical modeling to study the vibration analysis of cracked micro beams based on the MCST}

\section{Abbas $\operatorname{Rahi}^{1}$ (])}

Published online: 11 April 2018

(C) Springer-Verlag GmbH Germany, part of Springer Nature 2018

\section{Correction to: Microsystem Technologies}

https://doi.org/10.1007/s00542-018-3768-7

In the original publication of this article, the Eqs. (20), (21), (52), (54), (55), (56) and Figs. 4-15 were incorrectly published. The author would like to correct them as follows:

The Eqs. (20), (21), (52), (54), (55), and (56) should be corrected as follows:

$$
\begin{aligned}
& U_{c}=\frac{\left(1-\vartheta^{2}\right) b h}{E} \int_{0}^{\eta}\left(K_{I M}\right)^{2} d \eta \\
& C=\left[1+\frac{12}{(1+\vartheta)(1-\eta)^{2}}\left(\frac{l}{h}\right)^{2}\right]\left[\frac{\left(1-\vartheta^{2}\right) b h}{E} \frac{\partial^{2}}{\partial M^{2}} \int_{0}^{\eta}\left(K_{I M}\right)^{2} d \eta\right] \\
& \frac{d w_{2}}{d x}\left(L_{c}\right)-\frac{d w_{1}}{d x}\left(L_{c}\right)=\frac{d^{2} w_{1}}{d x^{2}}\left(L_{c}\right) \times \frac{S}{K_{t}}
\end{aligned}
$$

$$
\begin{gathered}
Q_{61}=\beta \cos \left(\beta L_{c}\right)-\frac{S \beta^{2}}{K_{t}} \sin \left(\beta L_{c}\right) ; \\
Q_{62}=-\beta \sin \left(\beta L_{c}\right)-\frac{S \beta^{2}}{K_{t}} \cos \left(\beta L_{c}\right) \\
Q_{63}=\beta \cosh \left(\beta L_{c}\right)+\frac{S \beta^{2}}{K_{t}} \sinh \left(\beta L_{c}\right) ; Q_{64} \\
=\beta \sinh \left(\beta L_{c}\right)+\frac{S \beta^{2}}{K_{t}} \cosh \left(\beta L_{c}\right) \\
C=\frac{\left(1-\vartheta^{2}\right) b h}{E} \frac{\partial^{2}}{\partial M^{2}} \int_{0}^{\eta}\left(K_{I M}\right)^{2} d \eta \\
K_{t}=\frac{1}{C}=\left[\frac{\left(1-\vartheta^{2}\right) b h}{E} \frac{\partial^{2}}{\partial M^{2}} \int_{0}^{\eta}\left(K_{I M}\right)^{2} d \eta\right]^{-1}
\end{gathered}
$$

Also, Figs. 4, 5, 6, 7, 8, 9, 10, 11, 12, 13, 14 and 15 should be corrected as follows:
The original article can be found online at https:// doi.org/10.1007/s00542-018-3768-7.

\section{Abbas Rahi}

a_rahi@sbu.ac.ir

1 Faculty of Mechanical and Energy Engineering, Shahid Beheshti University, A.C., Tehran, Iran 
Fig. 4 Torsional spring stiffness at the crack location of the microbeam with considering the SIF in model No. 1 for crack versus crack depth ratio $\eta=$ $a / h$ for different values of dimensionless material length scale parameter $l / h$

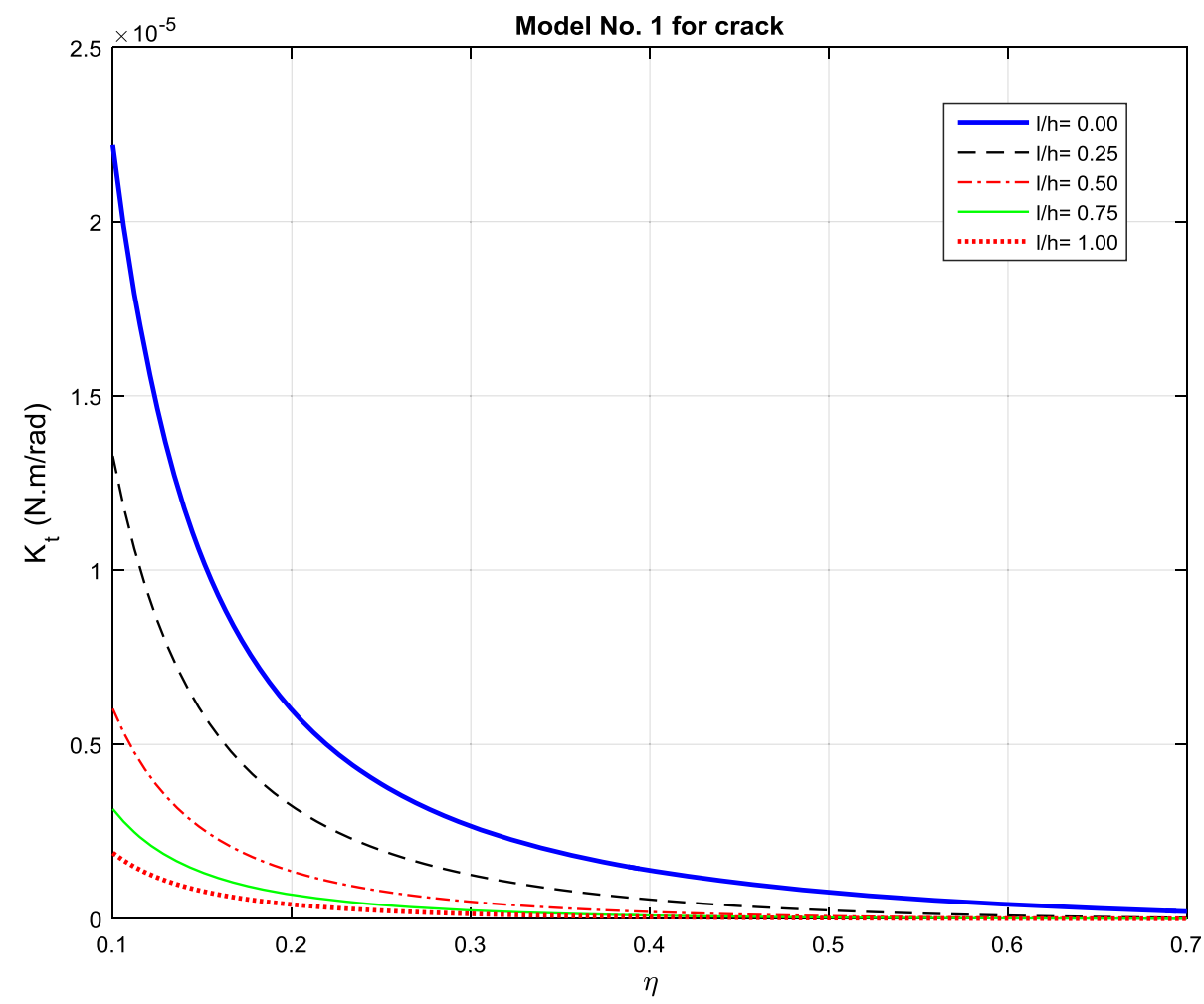

Fig. 5 Torsional spring stiffness at the crack location of the microbeam with considering the SIF in model No. 2 for crack versus crack depth ratio $\eta=$ $a / h$ for different values of dimensionless material length scale parameter $l / h$

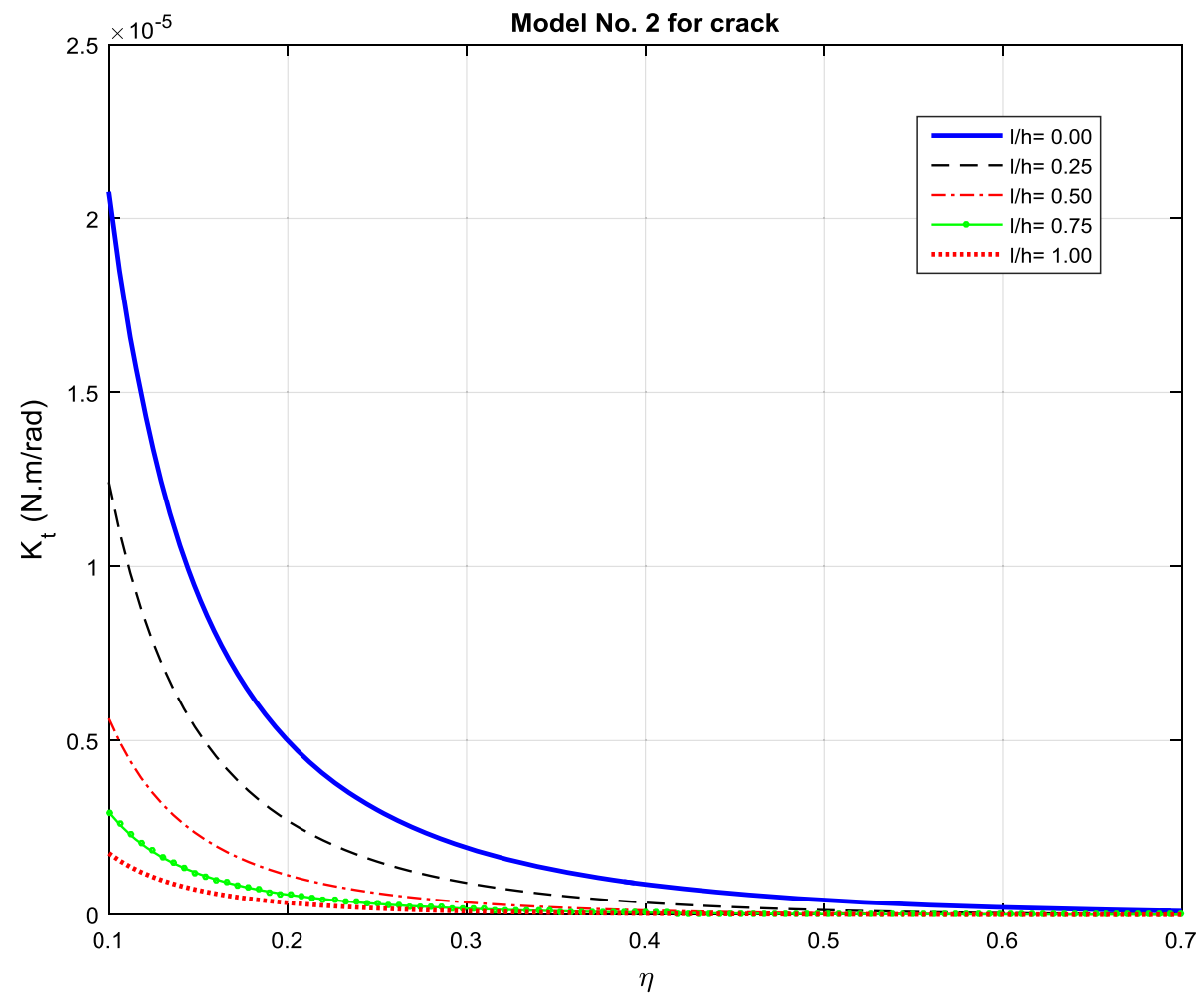


Fig. 6 Torsional spring stiffness at the crack location of the microbeam with considering the SIF in model No. 3 for crack versus crack depth ratio $\eta=$ $a / h$ for different values of dimensionless material length scale parameter $l / h$

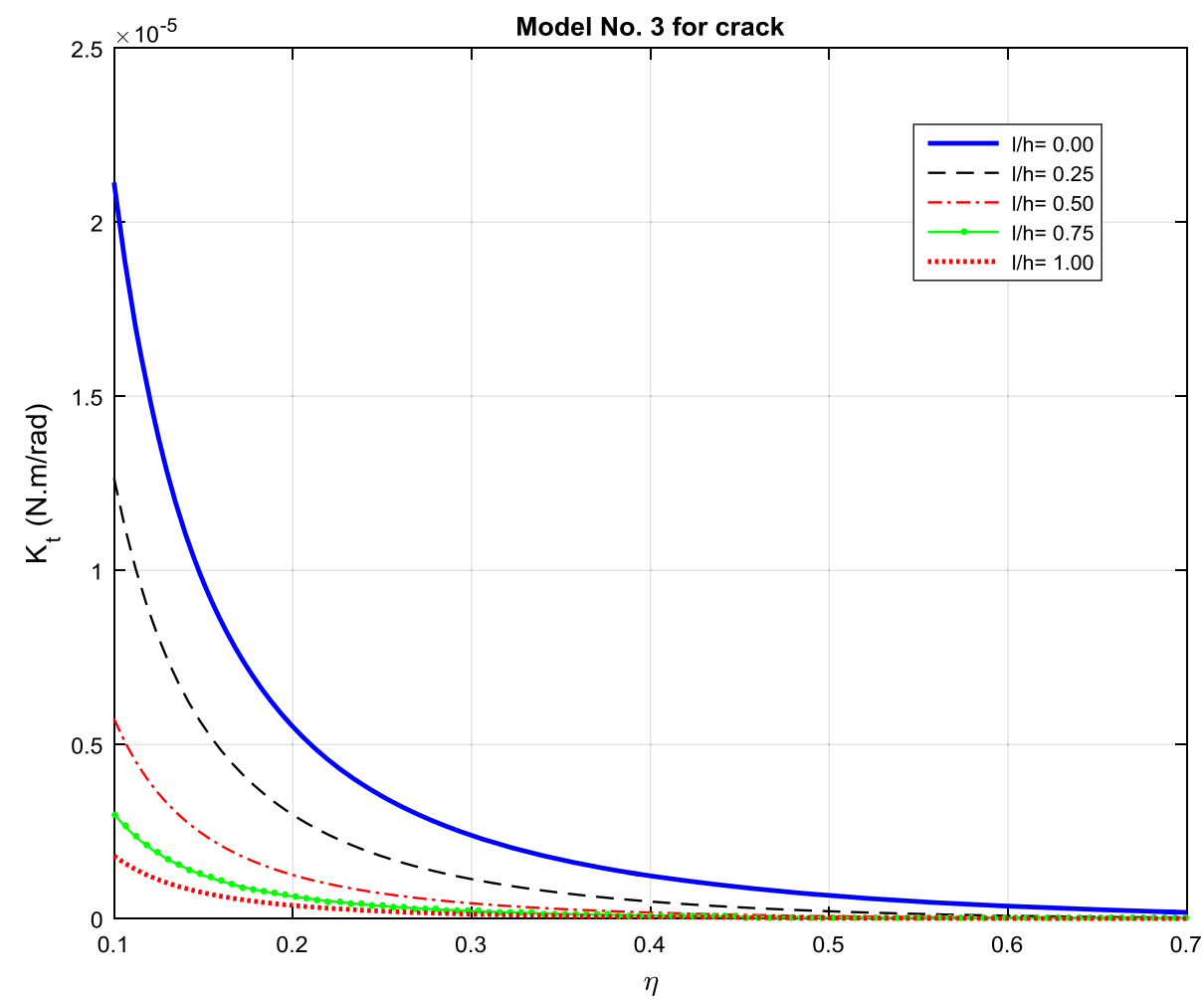

Fig. 7 Torsional spring stiffness at the crack location of the microbeam with considering the SIF in model No. 4 for crack versus crack depth ratio $\eta=$ $a / h$ for different values of dimensionless material length scale parameter $l / h$

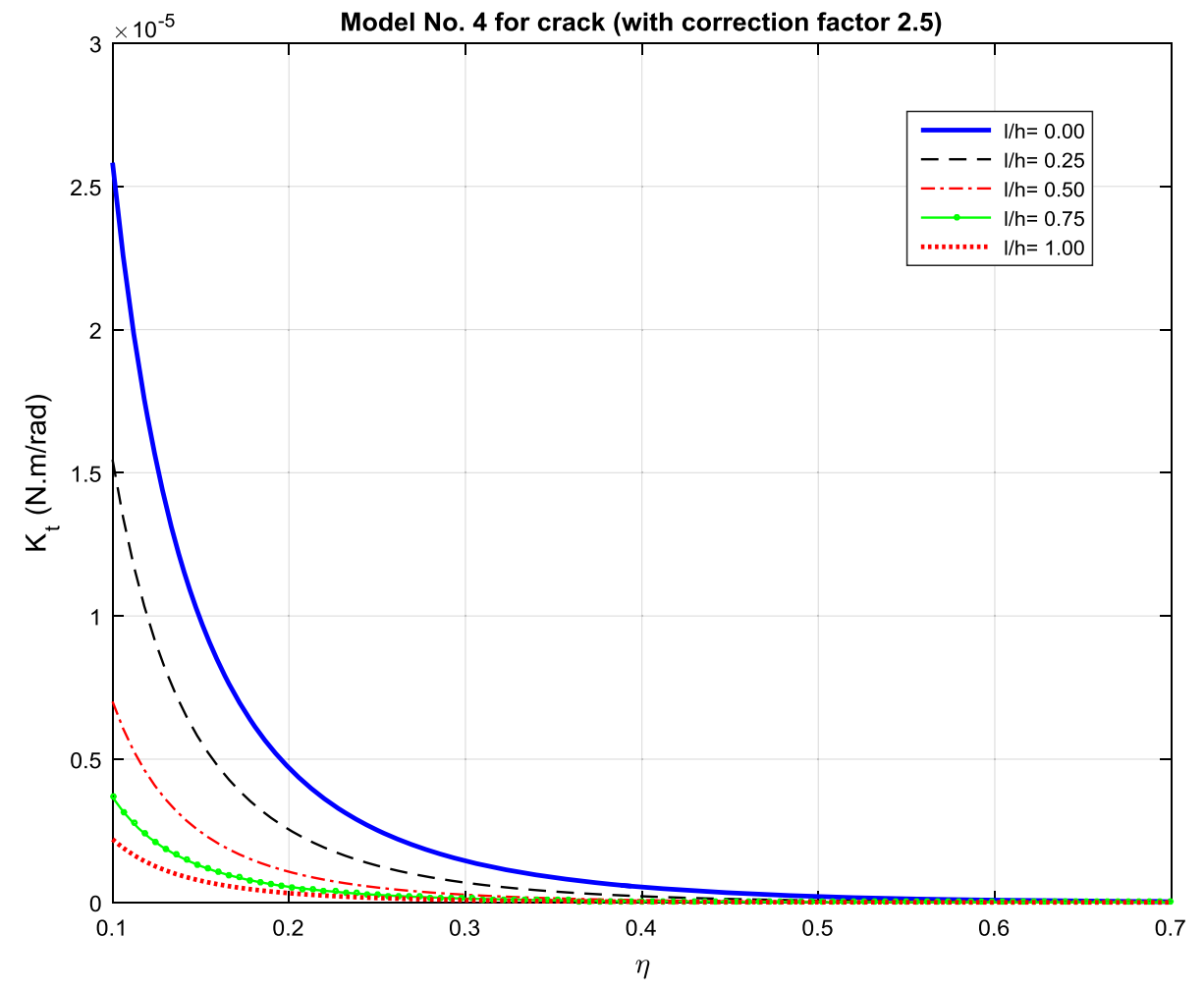


Fig. 8 Torsional spring stiffness at the crack location of the microbeam versus crack depth ratio $\eta=a / h$ for different models of crack at $\frac{l}{h}=0$

Fig. 9 Torsional spring stiffness at the crack location of the microbeam versus crack depth ratio $\eta=a / h$ for different models of crack at $\frac{l}{h}=0.5$
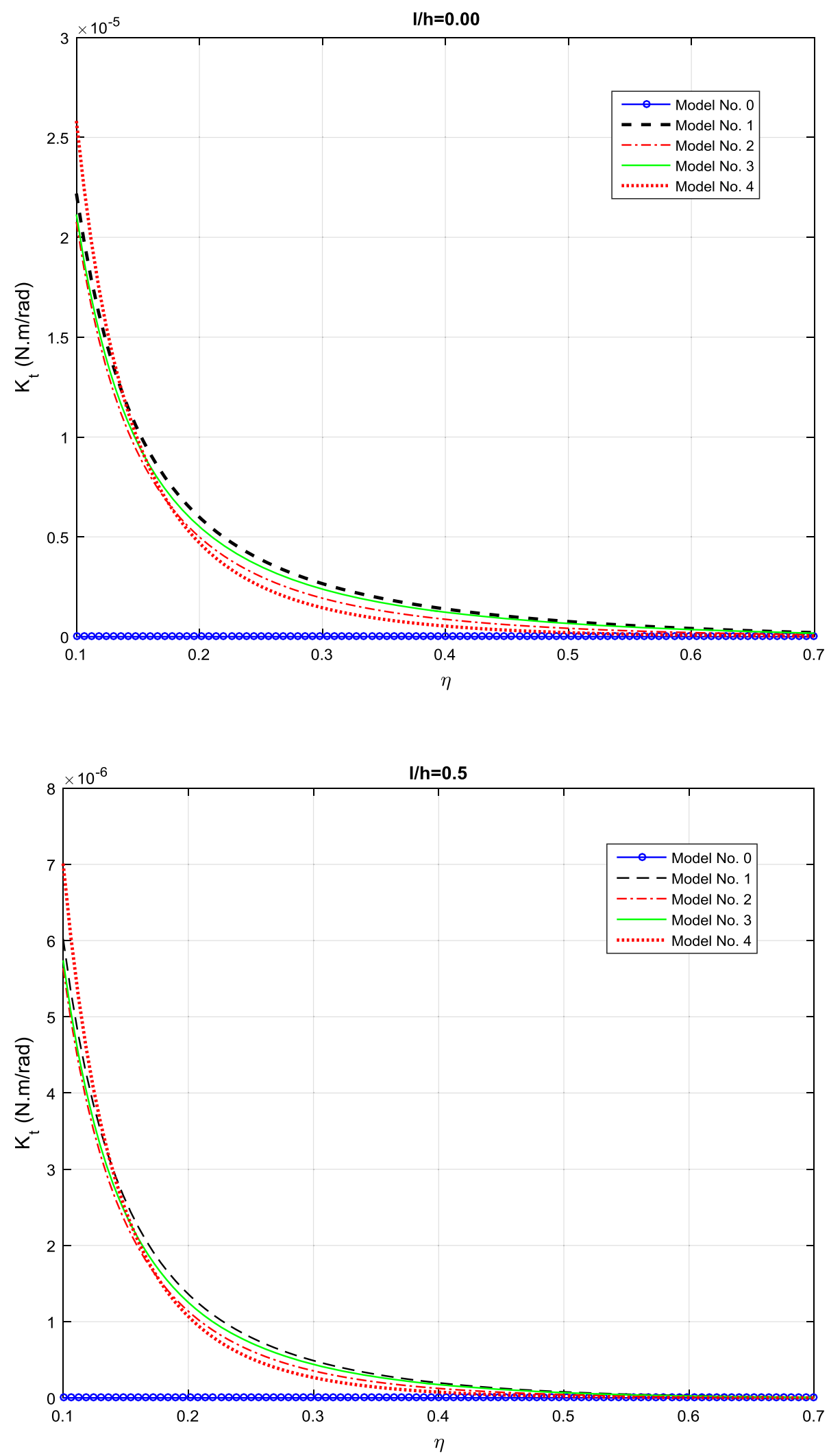
Fig. 10 Torsional spring stiffness at the crack location of the microbeam versus crack depth ratio $\eta=a / h$ for different models of crack at $\frac{l}{h}=1$

Fig. 11 Variation of fundamental natural frequency of the cracked microbeam versus crack depth ratio $\eta=$ $a / h$ for different crack location at $\frac{l}{h}=1$
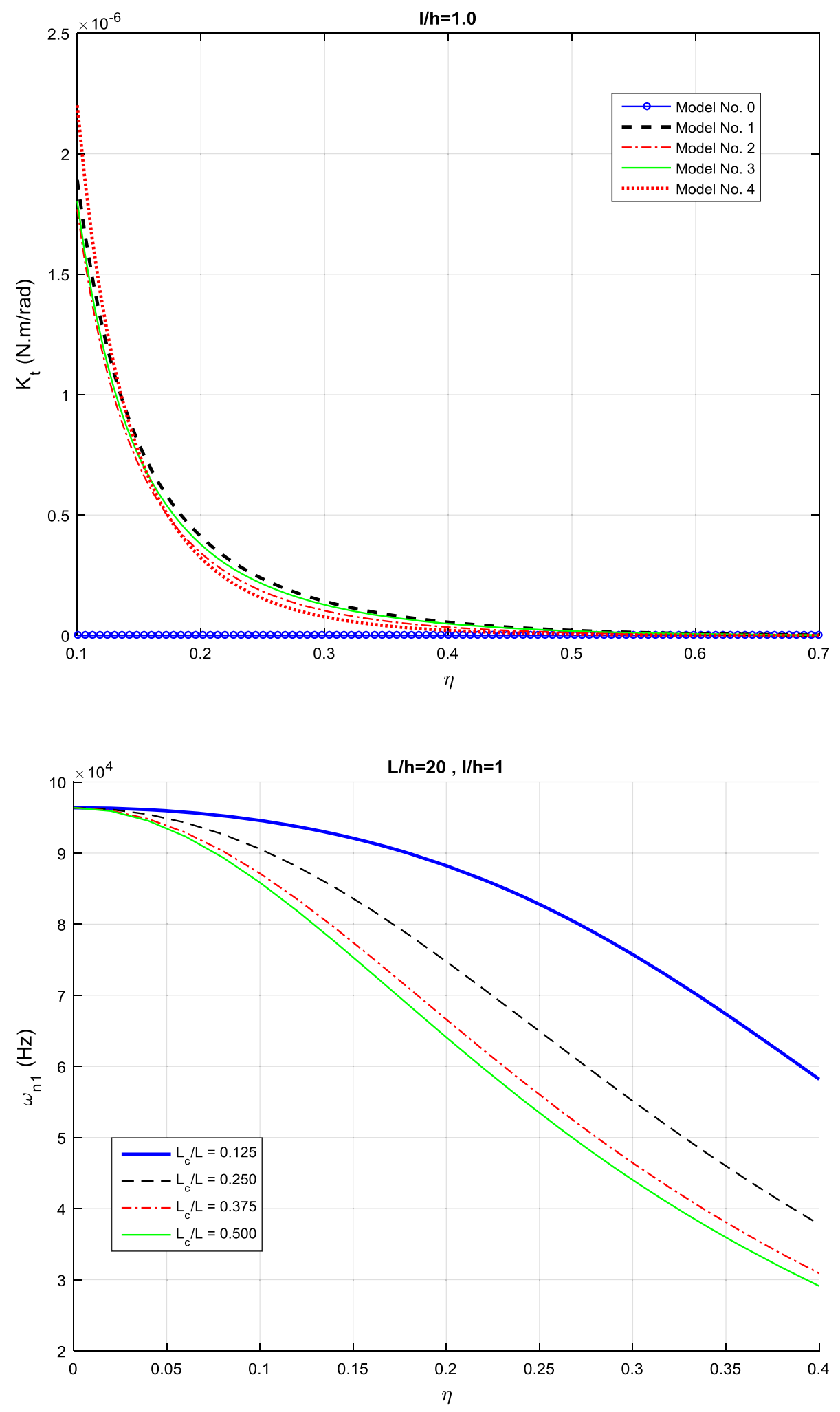
Fig. 12 Variation of second natural frequency of the cracked microbeam versus crack depth ratio $\eta=a / h$ for different crack location $\frac{L_{c}}{L}$ at $\frac{l}{h}=1$

Fig. 13 Variation of

fundamental natural frequency of the cracked microbeam versus crack depth ratio $\eta=$ $a / h$ for different values of dimensionless material length scale parameter $l / h$ at crack location $\frac{L_{c}}{L}=0.5$
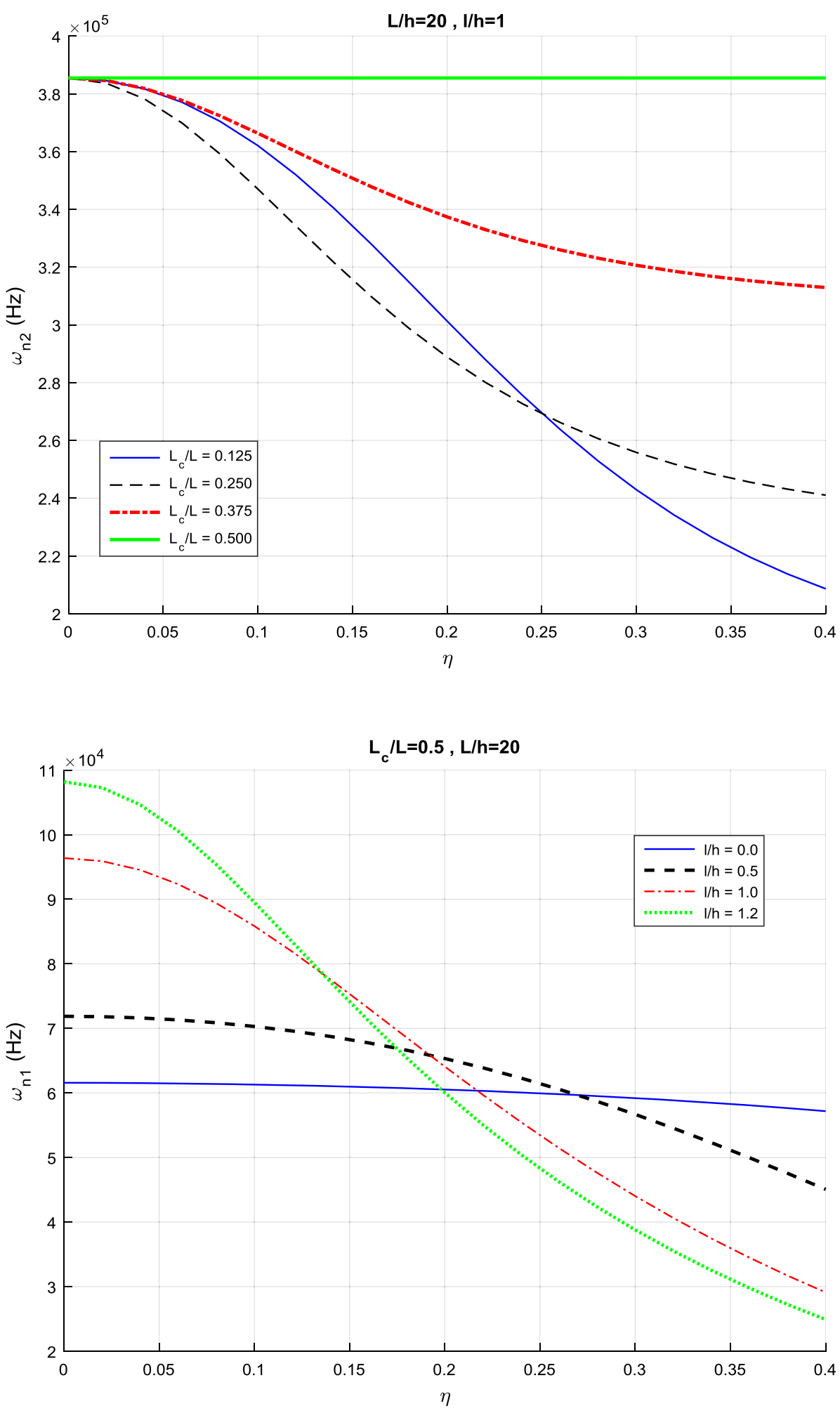
Fig. 14 Variation of fundamental natural frequency of the cracked microbeam versus crack location $\frac{L_{c}}{L}$ for different values of dimensionless material length scale parameter $l / h$ at crack depth ratio $\eta=0.2$
Fig. 15 Variation of second natural frequency of the cracked microbeam versus crack location $\frac{L_{c}}{L}$ for different values of dimensionless material length scale parameter $l / h$ at crack depth ratio $\eta=0.2$
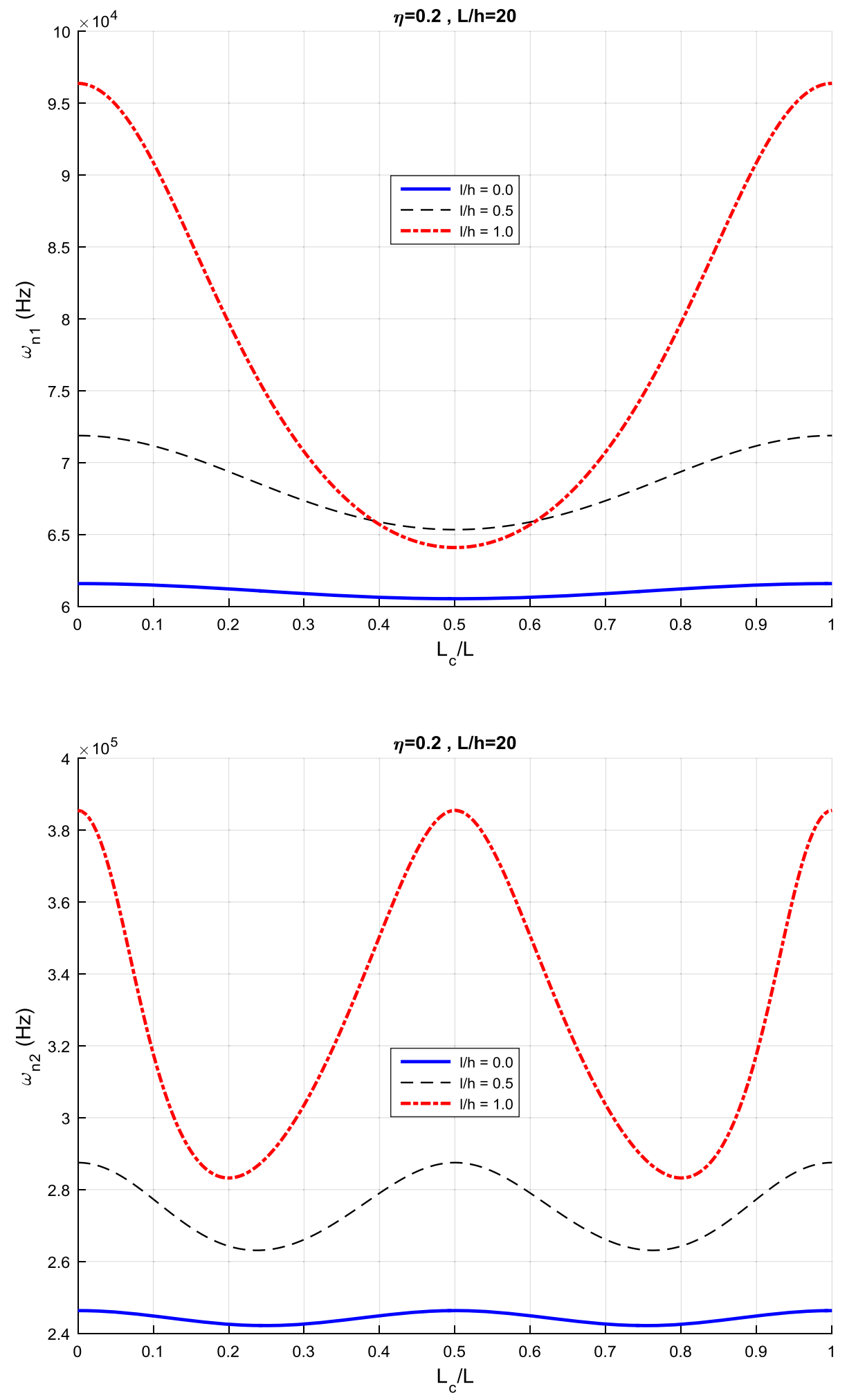